\title{
Erratum to: Acute function of secreted amyloid precursor protein fragment APPs $\alpha$ in synaptic plasticity
}

\author{
Meike Hick · Ulrike Herrmann • Sascha W. Weyer · Jan-Philipp Mallm • \\ Jakob-Andreas Tschäpe • Marianne Borgers • Marc Mercken • Fabian C. Roth • \\ Andreas Draguhn $\cdot$ Lutz Slomianka $\cdot$ David P. Wolfer $\cdot$ Martin Korte $\cdot$ Ulrike C. Müller
}

Published online: 20 December 2014

(C) Springer-Verlag Berlin Heidelberg 2014

\section{Erratum to: Acta Neuropathol}

DOI 10.1007/s00401-014-1368-x

Due to a typesetting error, the original version of this article contained a mistake which has been corrected. In Fig. 3a, the dendritic tracing was inadvertently cut off for the third neuron of NexCre cDKO mice (panel a, right border). Tracings of all other neurons are displayed correctly.

The correct version of Fig. 3 is given below.

The online version of the original article can be found under doi:10.1007/s00401-014-1368-x.

M. Hick · S. W. Weyer · J.-P. Mallm · J.-A. Tschäpe ·

U. C. Müller $(\triangle)$

Department of Bioinformatics and Functional Genomics, Institute of Pharmacy and Molecular Biotechnology, Heidelberg

University, Im Neuenheimer Feld 364, 69120 Heidelberg, Germany

e-mail: u.mueller@urz.uni-heidelberg.de

\section{U. Herrmann · M. Korte}

Division of Cellular Neurobiology, Zoological Institute,

TU Braunschweig, Braunschweig, Germany

Present Address:

J.-P. Mallm

Bioquant, Heidelberg University, Research Group Genome

Organization \& Function, Heidelberg, Germany

Present Address:

J.-A. Tschäpe

Roche Diagnostics International, Rotkreuz, Switzerland
M. Borgers · M. Mercken

Neuroscience Therapeutic Area, Janssen Research

and Development, Turnhoutseweg 30, 2340 Beerse, Belgium

F. C. Roth · A. Draguhn

Institute of Physiology and Pathophysiology, Heidelberg

University, Heidelberg, Germany

L. Slomianka $\cdot$ D. P. Wolfer

Institute of Anatomy, University of Zurich, Zurich, Switzerland

D. P. Wolfer

Institute of Human Movement Sciences and Sport, ETH Zurich, Zurich, Switzerland

M. Korte

AG NIND, HZI, Braunschweig, Germany 

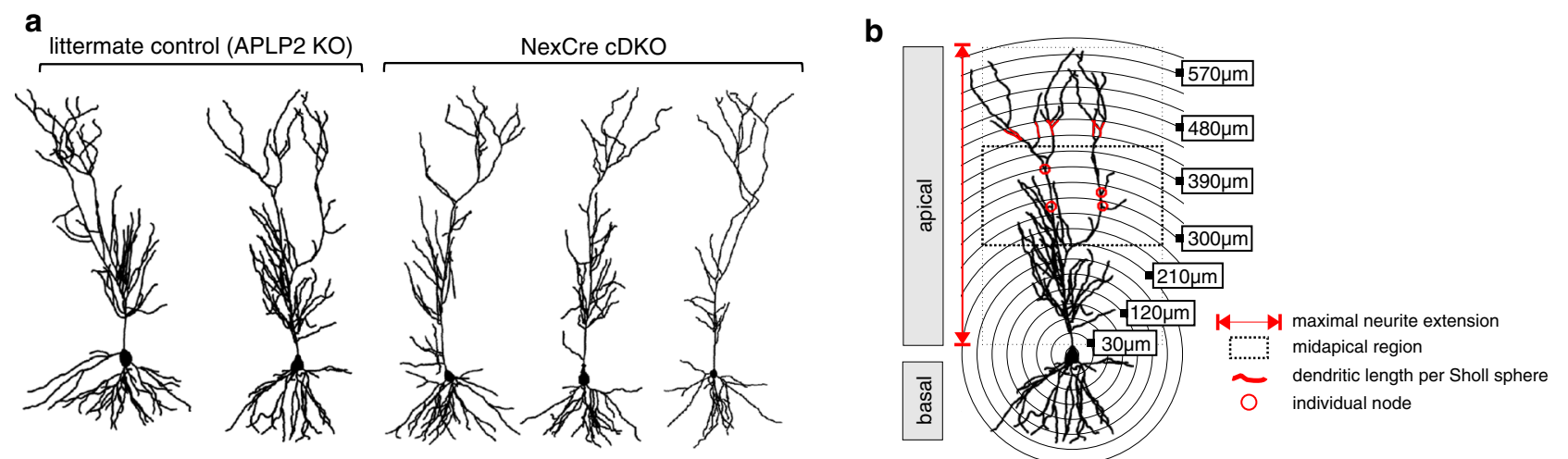
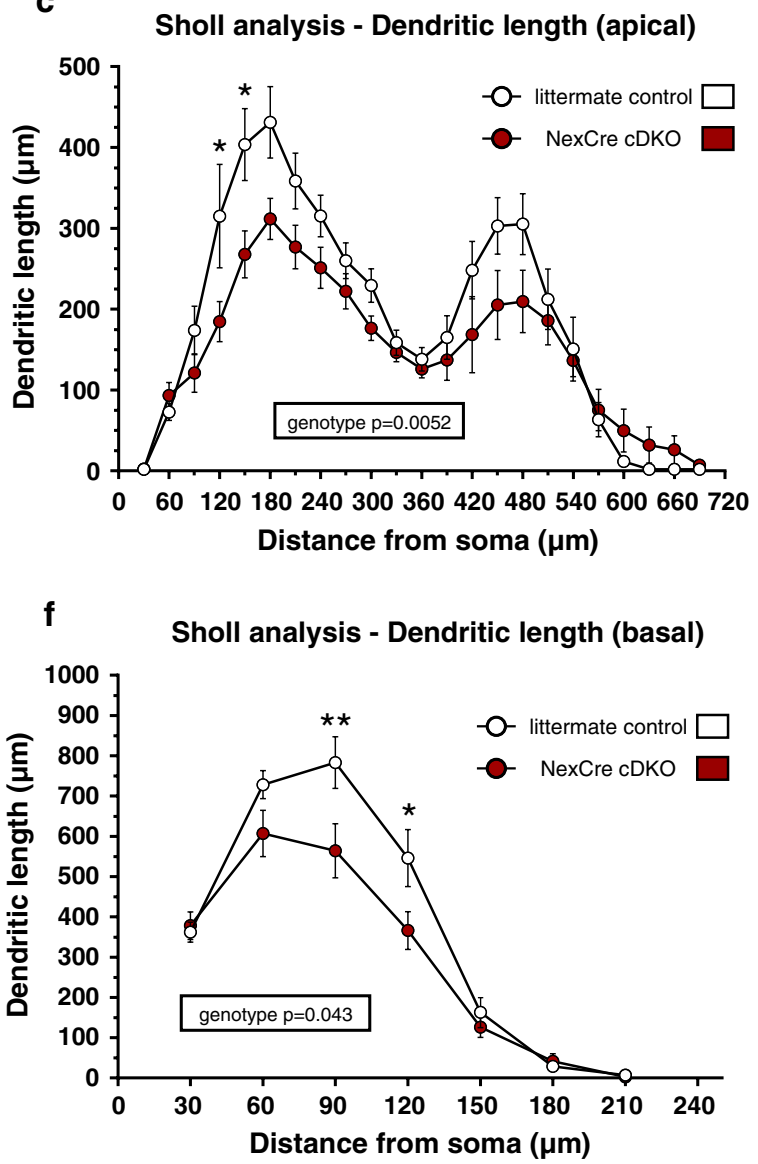

Fig. 3 Neurons of NexCre cDKO mice exhibit impaired dendritic complexity and reduced total neurite length. a Representative 3D-reconstructions of CA1 pyramidal neurons from littermate control (left) and NexCre cDKO mice (right). b Schematic representation of parameters assessed. c Sholl analysis reveals a significant overall genotype effect on apical dendritic morphology that was most prominent in proximal regions (repeated measures ANOVA: genotype $F(1,20)=9.818, p=0.0052$, with post hoc Bonferroni multiple comparison test, $\left.{ }^{*} p<0.05\right)$. d NexCre cDKO neurons display a signifi- d

Total dendritic length (apical)

e
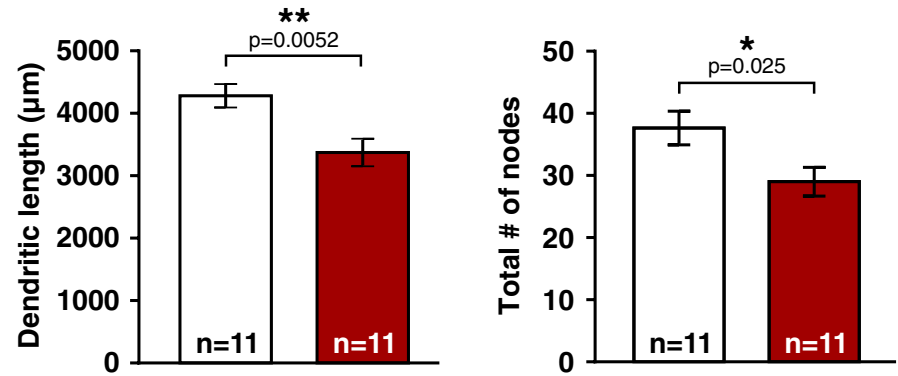

g

Total dendritic length (basal)

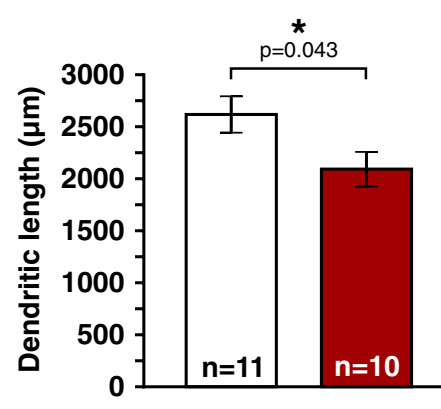

h Total dendritic branching (basal)

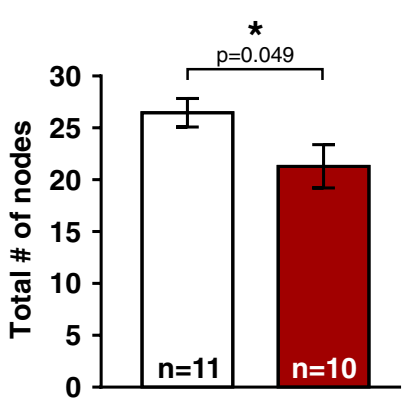

cantly reduced total apical dendritic length and e reduced dendritic branching. f Sholl analysis reveals a significant overall genotype effect on basal dendritic morphology (repeated measures ANOVA: genotype $F(1,19)=4.710, p=0.043$, with post hoc Bonferroni multiple comparison test, $\left.{ }^{*} p<0.05 * * p<0.01\right)$. g NexCre cDKO neurons display a significantly reduced total basal dendritic length and h dendritic branching. $n=$ number of neurons analyzed (from 5 animals/genotype, age: 11-13 weeks). Error bars SEM 